\title{
REDUCED-ORDER MODELS FOR THE CALCULATION OF THERMAL TRANSIENTS OF HEAT CONDUCTION/ CONVECTION FE MODELS
}

\author{
D. Botto, S. Zucca, and M. M. Gola \\ Department of Mechanical Engineering, Politecnico di Torino, C. so Duca degli Abruzzi, Torino, \\ Italy
}

\begin{abstract}
On-line calculation of temperature and thermal stresses at critical locations of structural components is currently performed in nuclear and aeronautical applications in order to assess fatigue damage accumulation and residual life. Since it is not possible to use full-scale finite element models because of the large calculation times, ad-hoc simplified algorithms are developed and employed. Thermal stresses commonly arise because of temperature gradients within the component due to heat exchange between the solid and the surrounding fluid. Commonly on-line monitoring methods are based on the assumption that time histories of the temperatures of the fluids around the component are known (e.g., measured) and are used as the inputs for the calculation of temperature and thermal stress at the critical locations of the component. If the temperatures of the surrounding fluid can not be measured, they must be numerically computed integrating an FE model of the fluid in the thermal FE model of the component; as a result, the size of the coupled thermal model is further increased. In the present work, a methodology is developed to reduce the size of thermal models including not only the FE model of the component but also the coupled fluid model. In detail, Guyan reduction and component mode synthesis are applied to a coupled thermal FE model, in order to reduce the size of the problem and to make it suitable for on-line calculations. The appropriate mathematical formulation for the reduction of the fluid FE model has been developed. Two reduction methods are proposed and are applied to the axial symmetric FE model of a turbine disk pointing out their capabilities and limitations for on-line temperature calculation.
\end{abstract}

Keywords: Coupled heat conduction/convection FE models; Reduced-order models; Reduction techniques; Thermal transient monitoring

\section{INTRODUCTION}

On-line calculation is currently used to evaluate temperature and thermal stresses of structures and machines characterized by high safety requirements and/or expensive maintenance costs (e.g., aircraft engines [1-3], nuclear power plants [4-6]) in order to assess fatigue damage accumulation and residual life. A typical full-scale thermo-mechanical FE model developed for fatigue damage

Address correspondence to S. Zucca, Department of Mechanical Engineering, Politecnico di Torino, C. so Duca degli Abruzzi 24, Torino 10129, Italy. E-mail: stefano.zucca@polito.it 
assessment is made of a solid FE model coupled to a fluid FE model by means of heat exchange convection coefficients.

In many cases, the fluid that surrounds the structural component flows along a path forming an advection network, where the term advection refers to the transport along a mass flow stream of some quantity, heat for instance, from one region to another. Only time history of the upstream fluid temperatures are known, while the time history of the fluid temperature along the network is governed by fluid/solid heat exchange phenomena.

A typical case is represented by turbine disks, where air flow is bled from the compressor exit, and it is used to cool the high and low pressure turbine disks: heat is transferred, by convection, from the disk boundary to the air flow stream. In the meantime, conduction occurs throughout the whole disk. The behavior of the coupled system air flow-disk can be simulated by means of a solid-fluid model coupled by heat exchange convection coefficients. In order to take into account this important feature in the design step, detailed fluid models of the advection network are included in the full-scale FE thermo-mechanical models.

Since it is neither possible nor necessary to perform on-line calculations on the whole component, a small number of areas, namely the critical locations, where a fatigue crack is expected to start, are identified by designers and selected for temperature and thermal stress monitoring. On-line calculations at critical locations cannot be performed with full-scale FE models, because of large memory requirements and expensive calculation times. Therefore reduced-order models are developed [1-9, 12-14] to compute only temperatures and thermal stresses at the critical locations of the component. The reference used to evaluate the accuracy of the reduced-order models are the temperatures and the thermal stresses evaluated by means of the full-scale thermo-mechanical FE models.

In the literature, several techniques to develop reduced-order models for temperature and thermal stress monitoring are proposed. In [1, 2], the constants of the equations used for temperature monitoring are obtained with a non-linear least-squares fitting over thermal transients computed by means of full-scale thermal FE models. In [4-6] Green's functions are computed off-line for both temperature and thermal stress monitoring in nuclear power plants are then used for on-line monitoring and fatigue damage assessment. In [7], the Green's functions approach is extended to thermal stress monitoring of non-linear systems.

In [8] non-linear identification techniques are employed to determine the matrix coefficients of a thermal system for temperature on-line calculations. In [9], linear identification techniques are successfully applied to develop reducedorder models for thermal stress monitoring. In the past, a method to reduce the degrees of freedom (DOFs) of a thermal Finite Element (FE) model, based on the well known techniques used in structural dynamics as Guyan reduction [10] and component modes synthesis (CMS) [11], has been proposed by the authors [12, 13] and applied to compute turbine disk thermal transients at critical locations. The resulting reduced-order model proved to be able to compute in short times the thermal transients in the critical location of the component. This methodology was then extended in [14] to compute temperatures and thermal stresses in non-linear applications. 
According to all these approaches, the boundary conditions of the reducedorder models are the time histories of all the fluid temperatures around the component, supposed to be known during the whole service life.

In the present article, the technique developed in [8] and [9] is extended in order to reduce not only the solid FE model of the structural component but also the fluid model of the advection network around it. As a consequence, the input of the reduced-order model will be the time histories of the upstream fluid temperatures, while temperatures along the fluid network are included in the set of the unknowns of the problem.

As a result, temperatures at the critical locations of the solid model will be computed directly from the time history of the upstream fluid temperatures of the advection network. Energy balance equations are first deduced for the advection network model, and coupling between advection and disk is discussed. Then the reduction techniques, developed in [10] and [11] from the structural dynamics and extended in [12-14] to thermal analysis, are described and two methods to extend these techniques to solid-fluid thermal FE models are proposed. Finally a study-case is presented: the coupled solid/fluid FE model of a turbine disk model surrounded by its secondary air system.

\section{FLUID MODEL}

In Figure 1 is depicted a simple FE model including both the solid model and the fluid model. Advection bars, representing the fluid flow, are drawn along the path followed by the fluid during its motion; nodes where advection bars link together are called fluid nodes. The fluid nodes and the advection bars constitutes the advection network. Each advection bar starts at fluid node $j$ with temperature $\mathrm{T}_{\mathrm{f}, \mathrm{j}}$, the upstream node, and ends up to fluid node $i$ with temperature $\mathrm{T}_{\mathrm{f}, \mathrm{i}}$, the downstream node. Mass flows from the upstream node to the downstream node.

For any fluid bar, the temperature at the downstream node does not affect the temperature at the upstream node.

Variation in the advection bar internal energy $\Delta \mathrm{Q}_{\mathrm{j}, \mathrm{i}}$, is proportional to the temperature difference between upstream and downstream node, according to the

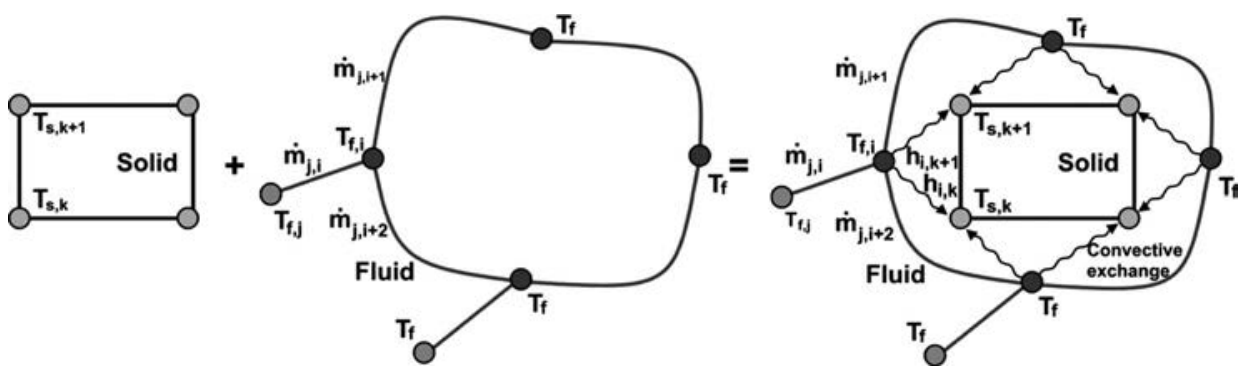

Figure 1 Example of a solid thermal model surrounded by the fluid model (advection network). 
following equation

$$
\Delta \mathrm{Q}_{j i}=\dot{\mathrm{m}}_{j i} \cdot \mathrm{c}_{\mathrm{P}} \cdot\left(\mathrm{T}_{f, i}-\mathrm{T}_{f, j}\right)
$$

with $\dot{\mathrm{m}}_{j i}$ : mass flow and $c_{p}$ : specific heat of the fluid.

Energy difference described by Eq. (1) is available for convection between advection bar and metal throughout the nodes lying on the boundary of the solid model. Hence, for each fluid node, the following balance energy equation holds

$$
\sum_{j=1}^{N_{A}} \dot{m}_{j i} c_{P}\left(T_{f, i}-T_{f, j}\right)+\sum_{k=1}^{N_{S}} h_{i k}\left(T_{f, i}-T_{s, k}\right)=0
$$

where $N_{A}$ is the number of advection bars entering the $i$ th fluid node and $N_{S}$ is the number of nodes with temperature $T_{s, k}$ at the solid model boundary, linked by convection to the $i$ th fluid node. The term $h_{i, k}$ is the heat transfer convection coefficient between the fluid node $i$ and solid node $k$. Eq. (2) is a steady-state equation, as fluid thermal inertia has been neglected. Eq. (2) can be written in matrix form as

$$
\mathbf{A D V} \cdot \mathbf{T}_{f}+\mathbf{H}_{f f} \cdot \mathbf{T}_{f}-\mathbf{H}_{f s} \cdot \mathbf{T}_{s}=0
$$

where ADV is the advection matrix collecting the mass flow terms, $\mathbf{H}_{\mathrm{ff}}$ and $\mathbf{H}_{\mathrm{fs}}$ are convection matrices collecting the convective contributes pertaining to the fluid and solid nodes, respectively; $\mathbf{T}_{\mathbf{s}}$ and $\mathbf{T}_{\mathbf{f}}$ are vectors collecting solid and fluid node temperatures, respectively. $\mathbf{A D V}$ and $\mathbf{H}_{\mathrm{ff}}$ are square matrices, with dimension $F \times F$, being $F$ the number of fluid nodes. The matrix $\mathbf{H}_{\mathrm{ff}}$ is diagonal. $\mathbf{H}_{\mathrm{fs}}$ is a rectangular matrix with dimension $\mathrm{F} \times S$, being $S$ the number of solid nodes. Diagonal elements in matrix $\mathbf{H}_{\mathrm{ff}}$, to be denoted $h f f$, $_{i i}$, are the sum of the elements on the corresponding row in matrix $\mathbf{H}_{\mathbf{f s}}$, to be denoted $h f s_{i j}$, namely

$$
h f f_{i i}=\sum_{j=1}^{F} h f s_{i j}
$$

\section{SOLID THERMAL MODEL}

Assembly of a thermal FE model is a task already performed in the literature. The resultant matrix equation of a general thermal problem is the following

$$
\mathbf{C} \cdot \dot{\mathbf{T}}_{s}+\overline{\mathbf{K}} \cdot \mathbf{T}_{s}=\mathbf{H}_{s g} \cdot \mathbf{T}_{g}-\mathbf{H}_{s s} \cdot \mathbf{T}_{s}
$$

where $\overline{\mathbf{K}}$ and $\mathbf{C}$ are the thermal conductivity and capacity matrix, respectively, $\mathbf{H}_{\mathrm{ss}}$ and $\mathbf{H}_{\mathbf{s f}}$ are the solid and fluid convection matrix collecting the convective terms pertaining to the solid and fluid nodes, respectively. The matrices $\overline{\mathbf{K}}, \mathbf{C}$ and $\mathbf{H}_{\mathrm{ss}}$ are square symmetric, with dimension $S \times S$. The matrix $\mathbf{H}_{\mathrm{ss}}$ is diagonal. $\mathbf{H}_{\mathrm{sf}}$ is a rectangular matrix, with dimension $S \times G$. The column vectors $\mathbf{T}_{\mathbf{s}}$ and $\mathbf{T}_{\mathbf{f}}$ contain solid and fluid temperatures, respectively.

Eq. (5) can be re-written in the more usual form

$$
\mathbf{C} \cdot \dot{\mathbf{T}}_{s}+\mathbf{K} \cdot \mathbf{T}_{s}=\mathbf{H}_{s f} \cdot \mathbf{T}_{f}
$$


where the matrix

$$
\mathbf{K}=\overline{\mathbf{K}}+\mathbf{H}_{s s}
$$

is the constrained thermal conductivity matrix. Diagonal elements in matrix $\mathbf{H}_{\mathrm{ss}}$, to be denoted $h s s_{i i}$, are the sum of the elements on the corresponding row in matrix $\mathbf{H}_{\mathrm{sf}}$, namely

$$
h s s_{i i}=\sum_{j=1}^{G} h s f_{i j}
$$

It can be easily shown that this simple relation holds between convection matrixes

$$
\mathbf{H}_{s f}=\mathbf{H}_{f s}^{\mathrm{T}}
$$

\section{SOLVING THE COUPLED SOLID/FLUID SYSTEM}

In order to compute the thermal transients of the system, Eqs. (3) and (5) must be solved, provided that all the matrices included in those equations are defined and that time histories of the temperatures of upstream fluid nodes of the advection network are known. Hence these fluid temperature will be referred as input fluid temperatures $\mathbf{T}_{\mathrm{f}}^{\mathrm{i}}$ related to the corresponding input fluid nodes. The temperature of all the other fluid nodes will be part of the solution of the system and will be called unknown fluid temperatures denoted $\mathbf{T}_{\mathrm{f}}^{\mathrm{x}}$. system

Parting the fluid temperatures in Eq. (3) it is possible to write the following

$$
\left[\begin{array}{ll}
\mathbf{A}^{i, i} & \mathbf{A}^{i, x} \\
\mathbf{A}^{x, i} & \mathbf{A}^{x, x}
\end{array}\right] \cdot\left\{\begin{array}{l}
\mathbf{T}_{f}^{i} \\
\mathbf{T}_{f}^{x}
\end{array}\right\}-\left\{\begin{array}{l}
\mathbf{H}_{f s}^{i} \\
\mathbf{H}_{f s}^{x}
\end{array}\right\} \cdot \mathbf{T}_{s}=\mathbf{0}
$$

where

$$
\mathbf{A}=\mathbf{A D V}+\mathbf{H}_{f f}
$$

In a similar manner Eq. (6) can be rewritten as

$$
\mathbf{C} \cdot \dot{\mathbf{T}}_{s}+\mathbf{K} \cdot \mathbf{T}_{s}=\mathbf{H}_{s f}^{\mathbf{i}} \cdot \mathbf{T}_{f}^{\mathbf{i}}+\mathbf{H}_{s f}^{\mathbf{x}} \cdot \mathbf{T}_{f}^{\mathbf{x}}
$$

The second row in Eq. (10) gives the temperatures of the unknown fluid nodes

$$
\mathbf{T}_{f}^{x}=\mathbf{A}^{x, x^{-1}} \cdot\left[\mathbf{H}_{f s}^{x} \cdot \mathbf{T}_{s}-\mathbf{A D V} \mathbf{V}^{x, i} \cdot \mathbf{T}_{f}^{i}\right]
$$

reminding that $\mathrm{H}_{\mathrm{ff}}^{\mathrm{x}, \mathrm{i}}=0$. Finally substitution of Eq. (13) into Eq. (12) gives the final equation

$$
\mathbf{C} \cdot \dot{\mathbf{T}}_{s}+\mathbf{K}_{\mathbf{1}} \cdot \mathbf{T}_{s}=\mathbf{H}_{\mathbf{1}} \cdot \mathbf{T}_{f}^{\mathbf{i}}
$$


where the unknown solid temperature are expressed in terms of input fluid temperatures only, while the unknown fluid temperatures have been statically reduced, with

$$
\begin{gathered}
\mathbf{K}_{\mathbf{1}}=\mathbf{K}-\mathbf{A}^{x, x^{-1}} \cdot \mathbf{H}_{f s}^{x} \\
\mathbf{H}_{\mathbf{1}}=\mathbf{H}_{s f}^{\mathbf{i}}-\mathbf{H}_{s f}^{\mathbf{x}} \cdot \mathbf{A}^{x, x^{-1}} \cdot \mathbf{A D} \mathbf{V}^{x, i}
\end{gathered}
$$

Despite their similar mathematical structure there is a great difference between Eqs. (6) and (14), since matrix $\mathrm{K}_{1}$, obtained by static reduction of the unknown fluid temperature of the fluid model, is not a symmetric matrix. Non-symmetry is due to advection coefficients contained in the matrix ADV. Time integration of Eq. (14) allows computing the time histories of the solid temperature directly from the input fluid temperatures.

\section{REDUCTION TECHNIQUES}

The static reduction of the fluid network described previously does not allow obtaining a reduced order model suitable for on-line calculations, since the size of the system of Eq. (14) is equal to the size of the full-scale solid thermal model. The aims of the reduction techniques are:

1. To reduce as much as possible the number of DOFs of the solid thermal model, allowing fast and accurate calculation of temperatures at critical locations of the component.

2. To remove from the reduced-order model the unknown fluid temperatures, keeping only the time history of input fluid temperatures.

Therefore in this section further reduction techniques, exported from the structural analysis, are proposed to reduce considerably the size of the solid thermal model. Reduction techniques are based on the hypothesis that the behaviour of the system can be simulated by a linear superposition of shape vectors. The larger is the number of shape vectors, the more accurate is the resulting reduced-order model.

What characterizes reduction techniques from one another is the choice of the shape vectors, which represent the column of the reduction matrix applied to the full-scale model. When reduction techniques of structural dynamics are extended to thermal problems, shape vectors assume the meaning of temperature distributions over the solid model and the application of a given reduction matrix to a thermal model implies the hypothesis that temperature within the full-scale model can be represented as a linear superposition of the temperature distributions included as columns of the reduction matrix.

\section{Guyan Method}

The Guyan reduction method [10] is based on the hypothesis that the system behaviour can be simulated by a linear superposition of steady-state temperature distributions. The system DOFs, solid temperatures $\mathbf{T}_{\mathbf{s}}$ in this application, are 
split into active and omitted, to be denoted $\mathbf{T}_{\mathbf{s}, \mathbf{a}}$ and $\mathbf{T}_{\mathbf{s}, \mathbf{o}}$, respectively. Eq. (6) is partitioned as

$$
\left[\begin{array}{ll}
\mathbf{C}_{\mathrm{aa}} & \mathbf{C}_{\mathrm{ao}} \\
\mathbf{C}_{\mathrm{ao}} & \mathbf{C}_{\mathrm{oo}}
\end{array}\right] \cdot\left\{\begin{array}{l}
\dot{\mathbf{T}}_{s, \mathrm{a}} \\
\dot{\mathbf{T}}_{s, \mathrm{o}}
\end{array}\right\}+\left\{\begin{array}{ll}
\mathbf{K}_{\mathrm{aa}} & \mathbf{K}_{\mathrm{ao}} \\
\mathbf{K}_{\mathrm{oa}} & \mathbf{K}_{\mathrm{oo}}
\end{array}\right\} \cdot\left\{\begin{array}{l}
\mathbf{T}_{s, \mathrm{a}} \\
\mathbf{T}_{s, \mathrm{o}}
\end{array}\right\}=\left\{\begin{array}{l}
\mathbf{H}_{\mathrm{sf}, \mathrm{a}} \\
\mathbf{H}_{\mathrm{sf}, \mathrm{o}}
\end{array}\right\} \cdot \mathbf{T}_{f}
$$

Omitted temperatures $\mathbf{T}_{\mathbf{s , 0}}$ are computed as linear superposition of active temperatures $\mathbf{T}_{\mathbf{s}, \mathbf{a}}$, solving the steady-state system

$$
\mathbf{T}_{s, \mathrm{o}}=-\mathbf{K}_{\mathrm{oo}}^{-1} \cdot \mathbf{K}_{\mathrm{oa}} \cdot \mathbf{T}_{s, \mathrm{a}}=\mathbf{G}_{\mathrm{oa}} \cdot \mathbf{T}_{s, \mathrm{a}}
$$

Since the shape vectors, defined at Eq. (18), which characterize the Guyan reduction, represent steady-state temperature distributions, the resulting reduced-order model is able to simulate exactly the steady-states with the same accuracy of the reference full FE model but is inaccurate in the case of steep transients, where thermal capacity plays an important role.

\section{Component Mode Synthesis}

In order to improve the accuracy of the reduced-order model in computing the active temperatures during steep transients, further shape vectors must be included. If the Component Mode Synthesis, described in [11] is extended to solid thermal FE models, a suitable set of new shape vectors is represented by the modal coordinates of the omitted DOFs, obtained solving the eigenproblem

$$
\left(-\lambda \cdot \mathbf{C}_{\mathrm{oo}}+\mathbf{K}_{\mathrm{oo}}\right) \cdot \mathbf{T}_{s, \mathrm{o}}=\mathbf{0}
$$

Eigenvectors $\boldsymbol{\Phi}$, solution of Eq. (19), are used to perform the modal transformation

$$
\overline{\mathbf{T}}_{s, \mathbf{0}}=\Phi \cdot \eta_{s, \mathbf{0}}
$$

where the terms of vector $\boldsymbol{\eta}_{s, o}$ are the modal temperatures.

As a result of Eqs. (18) and (20) the final reduction is

$$
\left\{\begin{array}{c}
\mathbf{T}_{s, \mathbf{a}} \\
\mathbf{T}_{s, \mathbf{0}}
\end{array}\right\}=\left[\begin{array}{cc}
\mathbf{I} & \mathbf{0} \\
\mathbf{G}_{\mathbf{o a}} & \mathbf{\Phi}
\end{array}\right] \cdot\left\{\begin{array}{l}
\mathbf{T}_{s, \mathbf{a}} \\
\boldsymbol{\eta}_{s, \mathbf{0}}
\end{array}\right\}=\boldsymbol{\Psi} \cdot\left\{\begin{array}{l}
\mathbf{T}_{s, \mathbf{a}} \\
\boldsymbol{\eta}_{s, \mathbf{0}}
\end{array}\right\}
$$

Equation (21) is a coordinate transformation in which the omitted temperatures are substituted by a linear superposition of active nodal temperatures and omitted modal temperatures. A reduced system is derived replacing Eq. (21) into Eq. (17), and then pre-multiplying Eq. (17) by $\boldsymbol{\Psi}^{\mathrm{T}}$. The reduced-order system of the solid thermal model is

$$
\widetilde{\mathbf{C}} \cdot\left\{\begin{array}{l}
\dot{\mathbf{T}}_{s, \mathrm{a}} \\
\dot{\boldsymbol{\eta}}_{s, \mathrm{o}}
\end{array}\right\}+\widetilde{\mathbf{K}} \cdot\left\{\begin{array}{l}
\mathbf{T}_{s, \mathrm{a}} \\
\boldsymbol{\eta}_{s, \mathrm{o}}
\end{array}\right\}=\widetilde{\mathbf{H}}_{\mathrm{sf}} \cdot \mathbf{T}_{f}
$$


where

$$
\begin{aligned}
\widetilde{\mathbf{C}} & =\boldsymbol{\Psi}^{\mathrm{T}} \cdot \widetilde{\mathbf{C}} \cdot \boldsymbol{\Psi} \\
\widetilde{\mathbf{K}} & =\boldsymbol{\Psi}^{\mathrm{T}} \cdot \mathbf{K} \cdot \boldsymbol{\Psi} \\
\widetilde{\mathbf{H}}_{s f} & =\boldsymbol{\Psi}^{\mathrm{T}} \cdot \mathbf{H}_{s f}
\end{aligned}
$$

If all the eigenvectors $\boldsymbol{\Psi}$ are used in Eq. (21), the size of the model of Eq. (22) is not reduced and the exact solution can be obtained for both steady-states and transients. Otherwise, if a few eigenvectors are selected, rejecting the others, the size of the model is reduced and solution is approximate. Guyan reduction can be seen as a particular case of the Component Mode Synthesis without modal temperatures.

\section{Guidelines for the Selection of Active and Omitted Temperatures}

In order to reduce a thermal FE model of a solid component the following guidelines are proposed:

a) The set of active temperatures must include the nodes lying at the critical locations of the component, since those temperatures represent the target of the thermal analysis.

b) The most important eigenvectors of omitted DOFs to be included in the reduced-order model, in order to improve accuracy of thermal transients, are those corresponding to the smallest eigenvalues. As a matter of fact, since the eigenvalues are the inverse values of the time constants of the thermal system, the eigenvectors corresponding to the smallest eigenvalues represent the temperature distributions with the largest decay times.

c) If the systems is made of several components connected to one another by interfaces, benefits in both accuracy and efficiency of the reduced-order model can be achieved by applying the sub-structuring technique [13]. According to this technique:

○ the system is divided in its elementary components;

o for each component a set of active temperatures is defined, including both nodes lying at the critical locations of the component and nodes lying at the component interfaces;

○ for each component a set of eigenvectors of omitted DOFs is defined, adopting the criterion described at point b);

o the reduced-order models of the elementary components are then assembled together by means of an assembly procedures typical of FE models. It must be noted that if nodes at interfaces are not included in the set of active temperatures of each component, final assembly is not possible.

d) If sub-structuring is adopted, it is possible to select more efficiently the eigenvectors of omitted DOFs. In fact, in order to improve the calculation of temperatures at critical locations lying at a given component, eigenvectors of omitted DOFs of that component must be first of all included in the reduced model. 


\section{COUPLED SOLID-FLUID MODEL REDUCTION}

The reduction techniques described in earlier have been already successfully applied to solid thermal FE models, in order to obtain reduced-order models for fast computation of temperatures $[12,13]$ and thermal stresses [14] at the critical locations of structural components. However, if these methods are applied directly to the balance equation system of Eq. (14) representing a coupled solid/fluid FE model, poor results are obtained. Indeed advection network contributions make matrix $\mathrm{K}_{1}$ non-symmetric. This implies that:

- the implementation of the Guyan reduction does not assure exact steady-state solutions,

- the eigenproblem of Eq. (19) may give complex eigenvectors with no physical meaning in thermal problems.

In order to exploit the capabilities of the reduction techniques without incurring physical nonsense, a different approach must be adopted. Here, two different approaches for coupled solid/fluid FE models are described.

\section{First Approach}

The approach described in this section has the aim of reducing statically the unknown fluid temperatures $\mathbf{T}_{f}^{x}$, obtaining a set of equations, characterised by symmetric coefficient matrices, similar to Eq. (14), to which the reduction techniques described earlier can be applied.

The procedure requires 3 steps:

1. The steady-state solid temperatures $\mathbf{T}_{s}$ are statically reduced from Eq. (12) as

$$
\mathbf{T}_{s}=\mathbf{K}^{-1} \cdot \mathbf{H}_{s f}^{i} \cdot \mathbf{T}_{f}^{i}+\mathbf{K}^{-1} \cdot \mathbf{H}_{s f}^{x} \cdot \mathbf{T}_{f}^{x}
$$

2. Equation (24) is replaced into fluid equilibrium Eq. (10) whose 2 nd row gives the unknown temperatures expressed in terms of the input fluid temperatures as

$$
\mathbf{T}_{f}^{x}=\mathbf{D}_{1}^{-1} \cdot \mathbf{D}_{2}^{-} \mathbf{T}_{f}^{i}
$$

where

$$
\begin{aligned}
& \mathbf{D}_{\mathbf{1}}=\mathbf{A}^{x, x}-\mathbf{H}_{f s}^{x} \cdot \mathbf{K}^{-1} \cdot \mathbf{H}_{s f}^{x} \\
& \mathbf{D}_{\mathbf{2}}=\mathbf{H}_{f s}^{x} \cdot \mathbf{K}^{-1} \cdot \mathbf{H}_{s f}^{i}-\mathbf{A}^{x, i}
\end{aligned}
$$

3. Finally Eq. (25) is replaced into Eq. (12) and the solving equation

$$
\mathbf{C} \cdot \dot{\mathbf{T}}_{s}+\mathbf{K} \cdot \mathbf{T}_{s}=\mathbf{H}_{\mathbf{1}} \cdot \mathbf{T}_{f}^{i}
$$

is obtained with

$$
\mathbf{H}_{\mathbf{1}}=\mathbf{H}_{s f}^{i}+\mathbf{H}_{s f}^{x} \cdot \mathbf{D}_{\mathbf{1}}^{-1} \cdot \mathbf{D}_{\mathbf{2}}
$$


By means of the above-described procedure, system coefficient matrices $\mathbf{C}$ and $\mathbf{K}$ have not been changed; therefore, the reduction methods described earlier can be directly applied to Eq. (27).

Equation (27) is afterward reduced by means of the methodologies described previously, since the left-hand side of the equation has not been modified by the preliminary steps. It becomes, for instance,

$$
\widetilde{\mathbf{C}} \cdot\left\{\begin{array}{l}
\dot{\mathbf{T}}_{s, \mathrm{a}} \\
\dot{\boldsymbol{\eta}}_{s, \mathrm{o}}
\end{array}\right\}+\widetilde{\mathbf{K}} \cdot\left\{\begin{array}{l}
\mathbf{T}_{s, \mathrm{a}} \\
\boldsymbol{\eta}_{s, \mathrm{o}}
\end{array}\right\}=\widetilde{\mathbf{H}}_{\mathbf{1}} \cdot \mathbf{T}_{f}^{i}
$$

where

$$
\widetilde{\mathbf{H}}_{1}=\boldsymbol{\Psi}^{\mathrm{T}} \cdot \mathbf{H}_{1}
$$

The fundamental hypothesis of the above-described reduction method is enclosed in Eq. (24), where the solid temperatures are supposed to be the steady-state values due to fluid temperatures neglecting the effect of thermal capacity.

The hypothesis is true in steady-state conditions but is an approximation during thermal transients. Therefore an exact steady-state solution is expected, while the error during transient is related to the number of eigenvectors included in the reduction matrix $\boldsymbol{\Psi}$. The approximation introduced by Eq. (24) cannot be completely overcome by eigenvectors of omitted DOFs of the system. Therefore thermal transients computed by means of the reduced-order model of Eq. (29) will tend towards thermal transients computed by means of the full scale model defined by Eq. (27) and not by that defined by Eq. (14).

\section{Second Approach}

In order to overcome the limitations outlined about the first reduction technique described before, a second approach is presented. The solid model is first of all reduced by means of the reduction methods described earlier as if the fluid network was not modelled. Then the reduction matrix used for the solid model is applied also to the fluid model. The generalized temperatures, defined according to Eq. (21), are replaced into the fluid balance equation (13) obtaining

$$
\mathbf{T}_{f}^{x}=\mathbf{A}^{x, x^{-1}} \cdot\left(\mathbf{H}_{f s}^{x} \cdot \boldsymbol{\Psi} \cdot\left\{\begin{array}{l}
\mathbf{T}_{s, \mathrm{a}} \\
\boldsymbol{\eta}_{s, \mathrm{o}}
\end{array}\right\}-\mathbf{A}^{x, i} \cdot \mathbf{T}_{f}^{i}\right)
$$

Eq. (22) is rewritten, parting the contributes of input and unknown fluid temperatures, as

$$
\widetilde{\mathbf{C}} \cdot\left\{\begin{array}{l}
\dot{\mathbf{T}}_{s, \mathrm{a}} \\
\dot{\boldsymbol{\eta}}_{s, \mathrm{o}}
\end{array}\right\}+\widetilde{\mathbf{K}} \cdot\left\{\begin{array}{l}
\mathbf{T}_{s, \mathrm{a}} \\
\boldsymbol{\eta}_{s, \mathrm{o}}
\end{array}\right\}=\widetilde{\mathbf{H}}_{s f}^{i} \cdot \mathbf{T}_{f}^{i}+\widetilde{\mathbf{H}}_{s f}^{x} \cdot \mathbf{T}_{f}^{x}
$$

Replacing Eq. (31) in Eq. (32) gives

$$
\widetilde{\mathbf{C}} \cdot\left\{\begin{array}{l}
\dot{\mathbf{T}}_{s, \mathrm{a}} \\
\dot{\boldsymbol{\eta}}_{s, \mathrm{o}}
\end{array}\right\}+\widetilde{\mathbf{K}}_{\mathbf{2}} \cdot\left\{\begin{array}{l}
\mathbf{T}_{s, \mathrm{a}} \\
\boldsymbol{\eta}_{s, \mathrm{o}}
\end{array}\right\}=\widetilde{\mathbf{H}}_{\mathbf{2}} \cdot \mathbf{T}_{f}^{i}
$$


where

$$
\begin{aligned}
\widetilde{\mathbf{K}}_{\mathbf{2}} & =\widetilde{\mathbf{K}}-\mathbf{H}_{s f}^{x} \cdot \mathbf{A}^{x, x^{-1}} \cdot \mathbf{H}_{f s}^{x} \cdot \boldsymbol{\Psi} \\
\widetilde{\mathbf{H}}_{\mathbf{2}} & =\mathbf{H}_{s f}^{i}-\mathbf{H}_{s f}^{x} \cdot \mathbf{A}^{x, x^{-1}} \cdot \mathbf{A}^{x, i}
\end{aligned}
$$

The reduced-order model of Eq. (33) does not allow computing exactly the steady-state temperatures at active nodes, since, in the fluid model equilibrium equation (31), the boundary solid temperatures are replaced with a linear superposition of generalized temperatures. Only if all the boundary solid temperatures are included in the set of active temperatures $\mathbf{T}_{\mathbf{s}, \mathbf{a}}$ then Eq. (31) would not contain any approximation leading to exact steady-state predictions.

With the above-described reduction technique, it is expected that the eigenvectors of omitted solid temperature affect both the transients and the steadystate values of the active temperatures. It is an important difference with respect to the first approach, described previously, 1, where steady-state temperatures at active nodes are predicted exactly and eigenvectors $\boldsymbol{\eta}_{\mathbf{s}, \mathbf{o}}$ only affect thermal transients. Furthermore if this approach is implemented, thermal transients computed by means of the reduced order model will tend towards the exact solution computed by means of the original full-scale FE model of Eq. (14).

\section{STUDY CASE: A TURBINE DISK MODEL}

\section{Model Description}

In this section the two reduction methods described before are applied to a study case, represented by the finite element model of a turbine disk. The model is made of:

(1) fluid FE model representing the Secondary Air System around the turbine disk; and (2) solid FE model representing the disk.

Figure 2 shows the diagram of a turbine Secondary Air System: cooling air is drawn off at the compressor exit; a portion of this flow is used to cool the high pressure turbine nozzle guide vanes; the remainder is used to cool blades and high and low pressure turbine disks.

Figure 3 shows a FE model of a turbine disk surrounded by the cooling air flow.

The FE thermal model of the disk is an axi-symmetric model made of triangular elements. The model includes a tailored mesh to govern the transport of mass, to simulate the cooling air flows. The total number of the model DOFs is about 2100.

Points numbered from 1 to 19 are fluid nodes and stand for chambers where flow has homogeneous conditions (temperature and pressure). The input fluid nodes are those numbered from 1 to 5 and 19; time histories of their temperature represent the boundary condition of the analysis. Fluid nodes 1-5 represent the upstream nodes of the Secondary Air System, while fluid node 19 represent the hot main flow. The unknown fluid nodes are instead those numbered from 6 to 18 .

In the FE model, the space coordinates of the fluid nodes are insignificant. To assure the correct convective coupling between the solid and fluid model it 


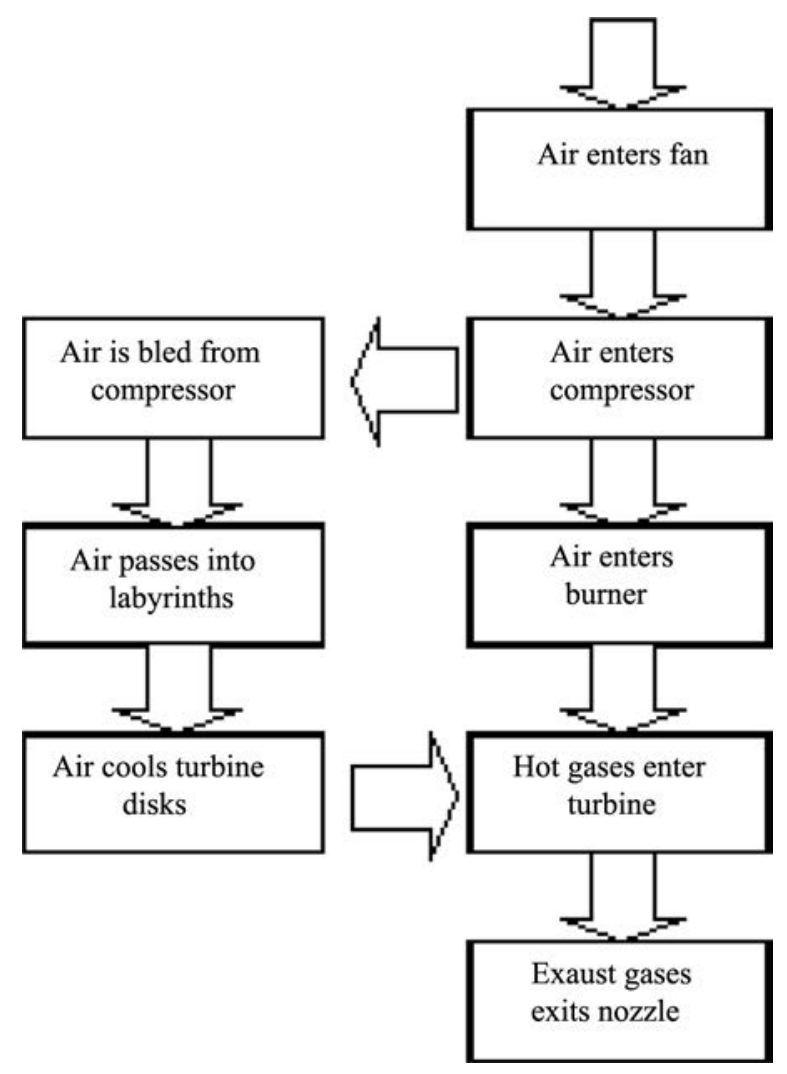

Figure 2 Simple diagram of a turbine cooling system.

is necessary that the fluid nodes are linked by means of convective heat transfer coefficients to the proper metal nodes on the disk boundary.

The convective coefficients used in the model have been calculated for each boundary portion of the disk. The model is characterized by constant convective coefficients, independent from spool speed and air flow temperature.

Flow parameters at the input fluid nodes $1-5$, namely temperature and mass flow, depend on the engine parameters, and derive from previous fluid dynamic calculations, not reported in this work.

Bars drawn around the disk, the advection bars, stand for the path followed by the cooling flow. The direction of the mass flow is pointed out by the arrows. The mass flow entering the system at the upstream nodes $1-5$ has been split among the advection bars according to fluid dynamic calculations.

The critical locations of the disk, whose temperature must be computed by the reduced-order models, are the points: A, the disk bore, B, the flange bolt, $\mathrm{C}$, the outer balancing area, and, D, the disk rim.

According to reduction techniques described previously, temperatures at critical areas A, B, C and D represent the set of active temperatures $\mathbf{T}_{\mathrm{s}, \mathrm{a}}$. All the other solid temperatures form the set of the omitted temperatures $\mathbf{T}_{\mathrm{s}, \mathrm{o}}$. 


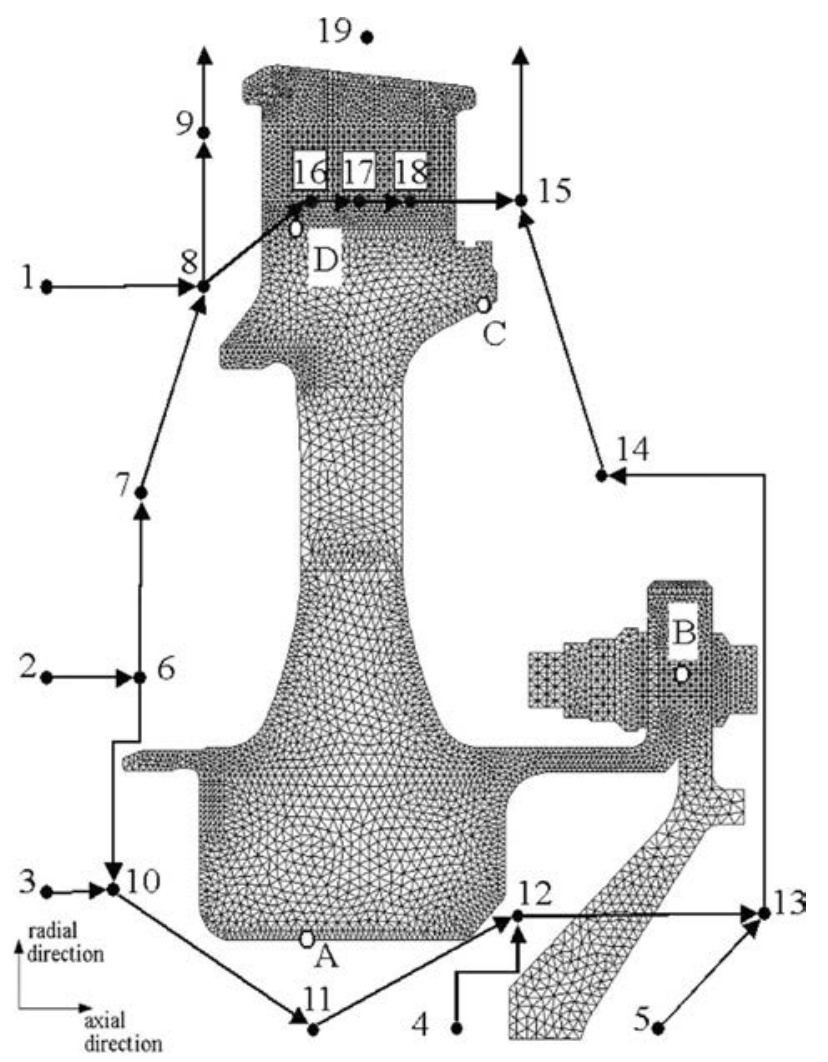

Figure 3 Disk FE thermal model: solid and fluid models.

\section{Tuning of the Reduced-Order Model to Reference Mission}

Once the active solid temperatures are defined, eigenvectors of omitted DOFs must be selected. The number of eigenvectors necessary to compute accurately the time history of the active solid temperatures cannot be estimated a priori, but it depends on the steepness of the thermal transients occurring in service. The steepest the thermal transients are, the larger is the number of eigenvectors to be included in the reduced-order model. To estimate the number of eigenvectors, it is here suggested to tune the reduced-order model to a reference mission having thermal transients comparable to those that will occur in the real mission profile.

In this case, the reference mission is the so-called "hot re-slam mission," largely employed in design to identify the critical locations of turbine disks. The time history of the spool speed and of two of the fluid input temperatures are plotted in Figures $4 \mathrm{a}$ and $4 \mathrm{~b}$, respectively.

The model tuning is performed by adding progressively the number of eigenvectors of the omitted DOFs of the solid model. The tuning procedure ends when the reduced-order model allows computing the time history of temperatures at active solid nodes with a maximum error lower than $10 \mathrm{~K}$ with respect to the full-scale FE model. 

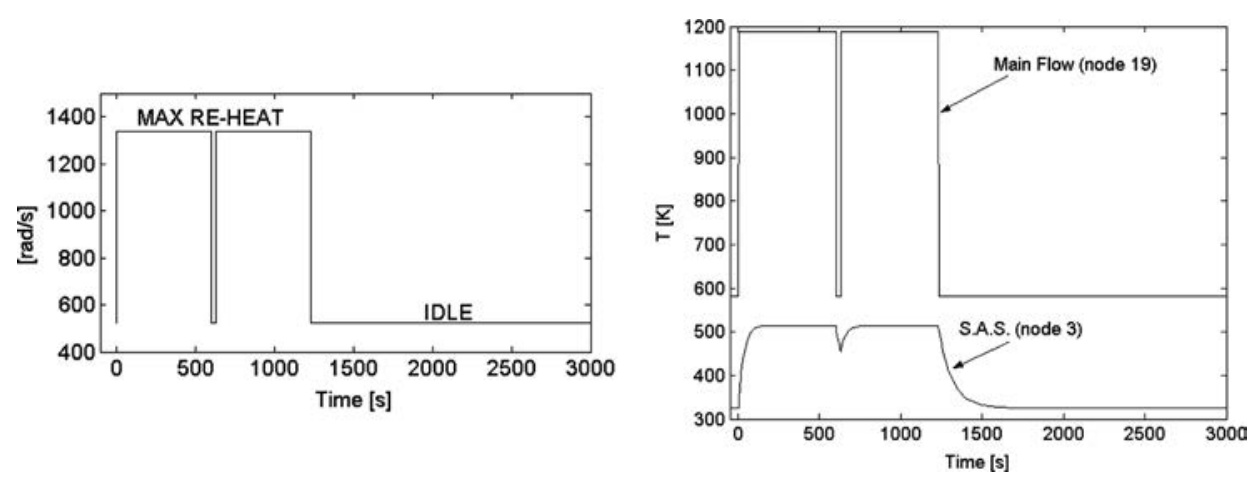

Figure 4 Hot re-slam mission profile: (a) spool speed; (b) upstream fluid temperatures 3 and 19.

In Figure 5, time history of active solid temperatures at point $\mathrm{A}$ and $\mathrm{C}$ are shown for both the approaches discussed before, having selected zero eigenvectors of omitted DOFs in both cases.

The plots of Figure 5 confirm the preliminary discussion of the proposed approaches written in Section 6:

1. If the first approach is implemented (Figure 5a), steady-state temperatures at active solid nodes are computed exactly (see the final temperature at $t=3000 \mathrm{~s}$ );

2. If the second approach is implemented (Figure 5b), steady-state temperatures at active solid nodes are not computed correctly (see the final temperature at $t=3000 \mathrm{~s})$;

3. In both cases, transients of active solid temperatures $\mathbf{T}_{s, a}$ are poorly estimated. In detail the reduced-order models, obtained implementing the Guyan reduction, under-estimate the time necessary to reach the steady-state temperature.

In order to understand the reasons of the features described at point 3 , it may be useful to start from structural dynamics. When reduction matrix $\boldsymbol{\Psi}$ is applied to a
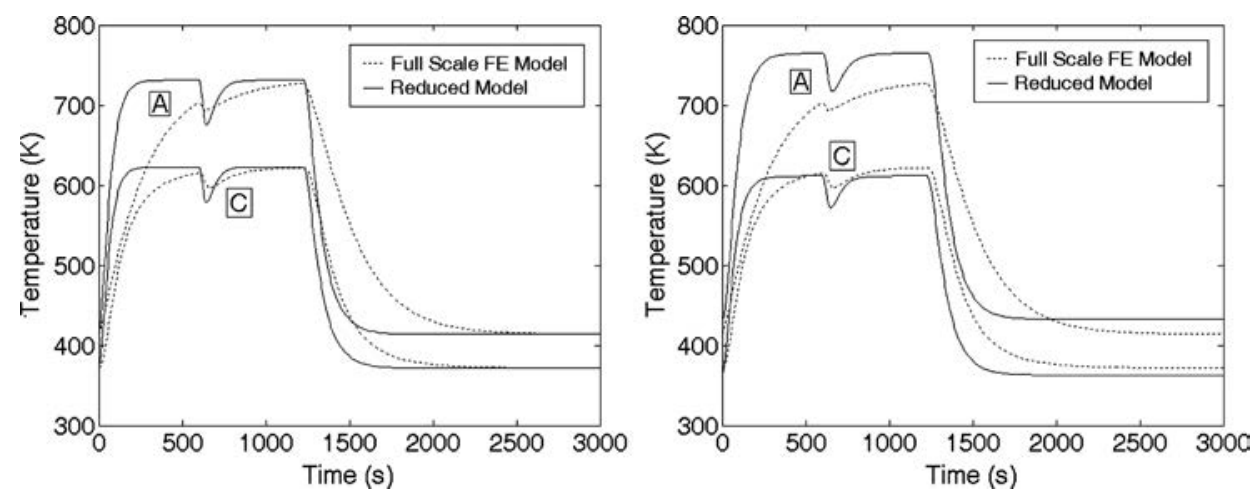

Figure 5 Time history of temperature at critical locations A and C during "hot re-slam mission." Comparison between full FE model and reduced model with 0 eigenvectors of omitted DOFs. (a) first approach; (b) second approach. 

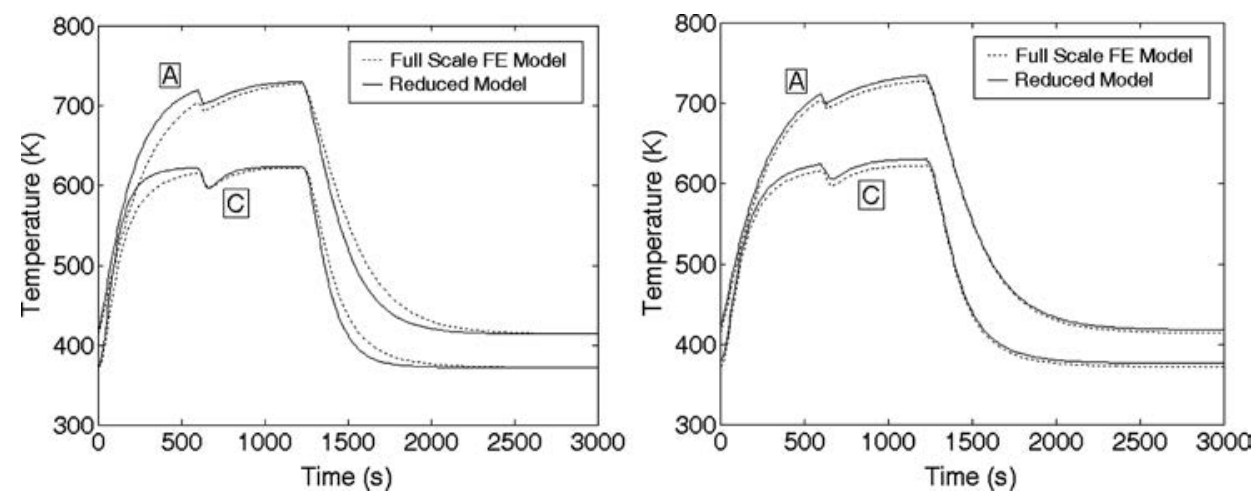

Figure 6 Time history of temperature at critical locations A and C during "hot re-slam mission." Comparison between full FE model and reduced model with 50 eigenvectors of omitted DOFs. (a) first approach; (b) second approach.

structural FE model, an assumption is made about the field of displacements of the model. Reducing the full-scale structural model corresponds to adding constraints. As a results, the eigenvalues of the reduced-order model, corresponding to its natural frequencies, are larger than the corresponding ones of the full-scale model.

The same occurs to reduced thermal models. In this case, the eigenvalues of the system represent the inverse values of the time constants of the thermal system. Therefore the transients of the reduced-order model vanish more quickly than those of the full-scale FE model.

To improve the accuracy of the reduced order-models, eigenvectors of omitted DOFs are computed by solving Eq. (19) and are progressively added to the reducedorder model. In Figure 6, there are the plots of the temperatures at critical locations $\mathrm{A}$ and $\mathrm{C}$ during the "hot re-slam mission" computed with reduced-order models characterized by 50 eigenvectors of omitted DOFs. In Figure 7, there are the plots of
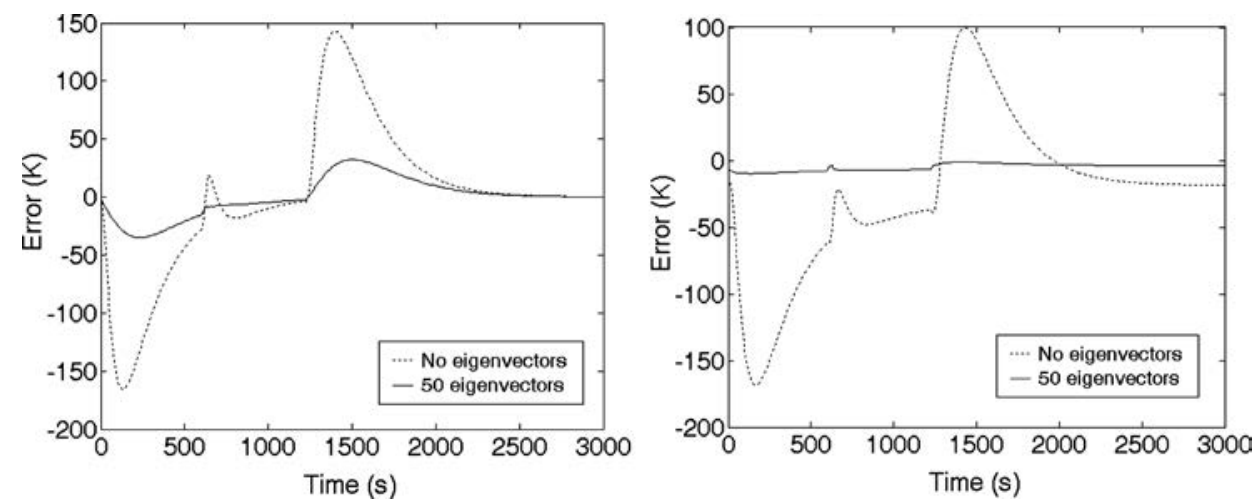

Figure 7 Error in the computation of temperature at critical location A during "hot re-slam mission." Comparison between full FE model and reduced model with 50 eigenvectors of omitted DOFs. (a) first approach; (b) second approach. 

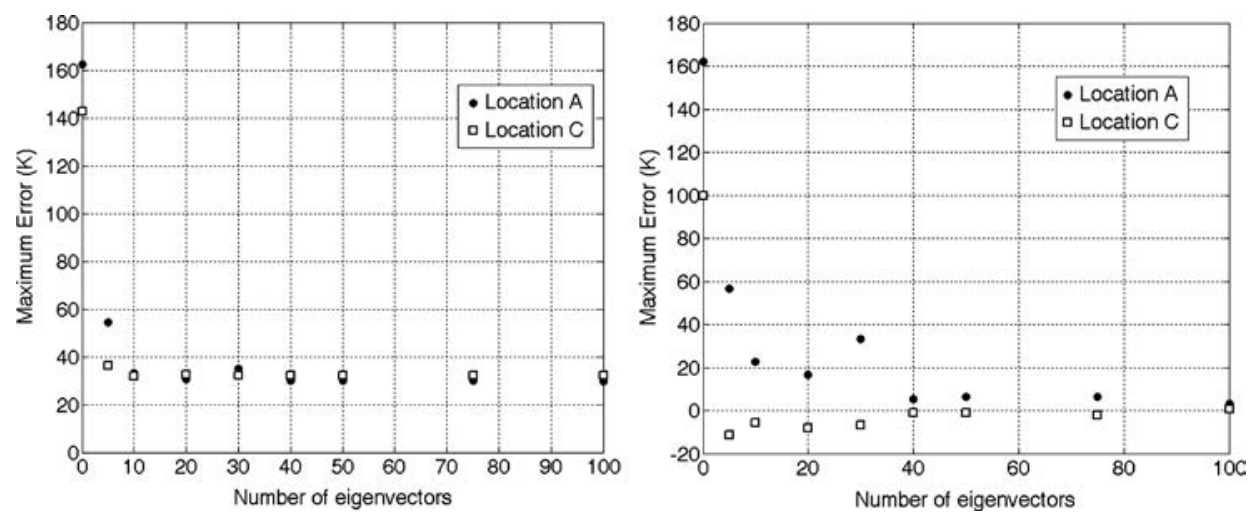

Figure 8 Maximum error in the computation of temperature at critical locations $\mathrm{A}$ and $\mathrm{C}$ during "hot re-slam mission." (a) first approach; (b) second approach.

the error made by the reduced models during the mission, expressed as the difference between the time histories of active temperatures computed by the original FE model of Eq. (14) and those computed by the reduced-order models. Finally in Figure 8, for both the presented approaches the maximum error in the time history of active temperatures is plotted against the number of eigenvectors included in the reduced model.

The following remarks can be made:

1. If the first approach is implemented (Figures $6 \mathrm{a}$ and 7a), steady-state temperatures at active nodes are computed exactly. A relevant error (about $30 \mathrm{~K}$ ) is still made during the transients. In accordance to what stated in Section 6.1, the reduced model obtained implementing the first approach will tend towards the solution of the system of Eq. (27) and not of the original full scale model of Eq. (14). In facts, as shown in Figure 8a, further increase in the number of eigenvectors $(>50)$ does not affect the accuracy of the reduced-order model.

2. If the second approach is implemented (Figures $5 \mathrm{~b}$ and $6 \mathrm{~b}$ ), steady-state temperatures at active solid nodes are not computed exactly, but error in both steady-states and transients decreases as eigenvectors of omitted DOFs are included in the reduced model. As shown in Figure 8b, further increase in the number of eigenvectors $(>50)$ would allow one to improve the accuracy of the reduced model, since the solution tends towards that computed by means of the full-scale original FE model of Eq. (14).

\section{Application of the Reduced-Order Model to a Real Mission}

A second mission profile, denoted as "real mission," has been used to verify the accuracy of the reduction methods. The time histories of the engine parameters of the real mission have been recorded during engine service [4]. The time history of the spool speed and of two of the fluid input temperatures are plotted in Figures 9a and $9 \mathrm{~b}$ respectively. 

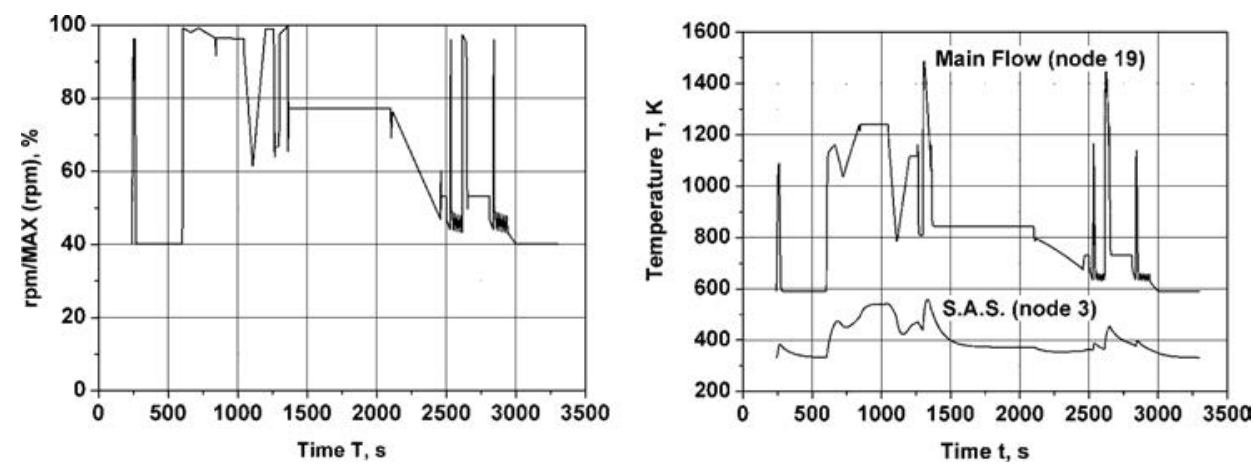

Figure 9 Real mission profile: (a) spool speed; (b) upstream fluid temperatures 3 and 19.
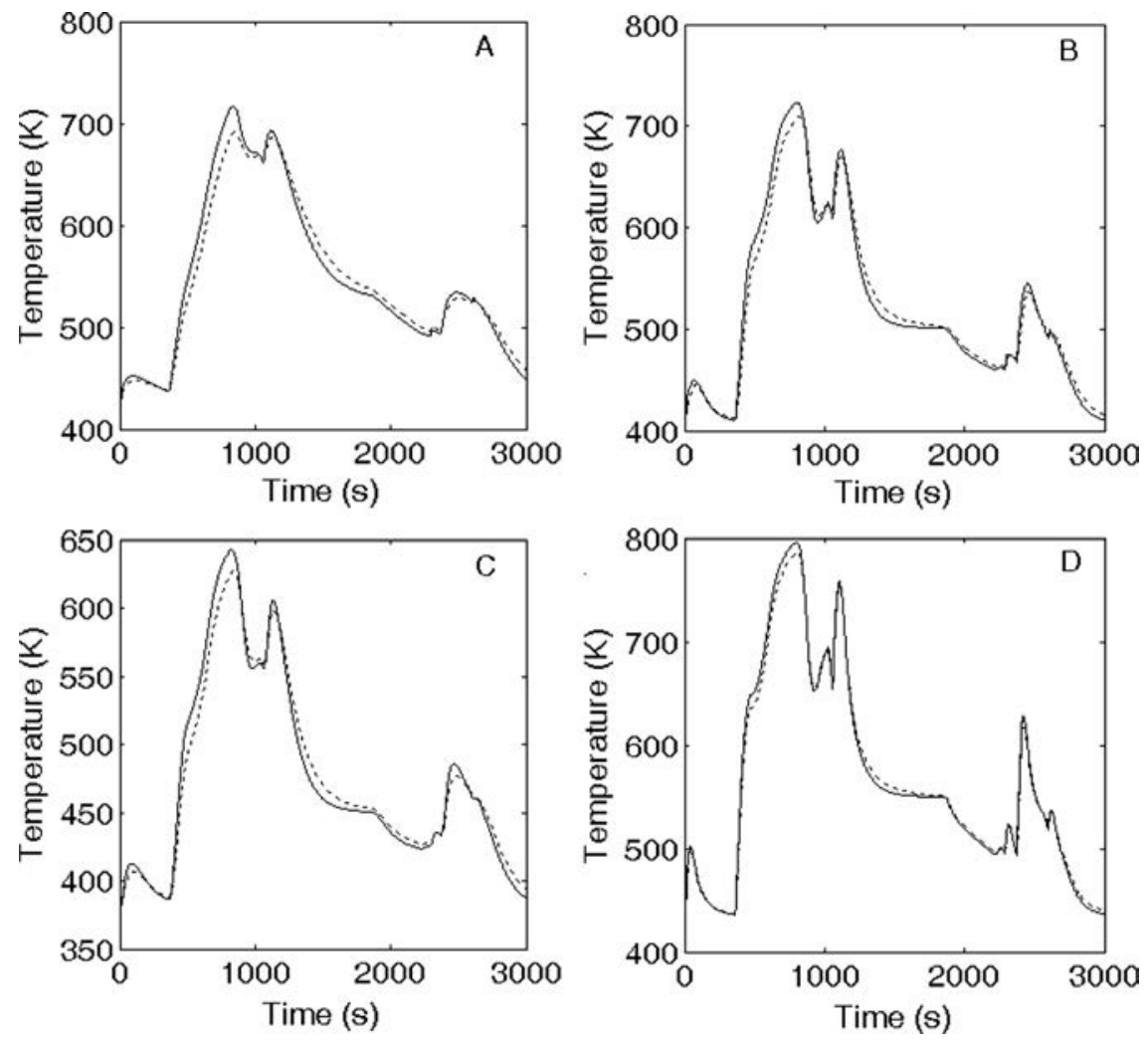

Figure 10 Time history of temperature at critical locations during "real mission." Comparison between full FE model (dotted lines) and reduced model (full lines) with 50 eigenvectors of omitted DOFs: first approach. 
The reduced models of the system made of 4 active temperatures and 50 eigenvectors used in the reference mission are employed. Time histories obtained by time integration of the reduced model are plotted in Figures 10 and in 11.

Also in this case, the error is represented by the difference between the time histories of active temperatures computed by the original FE model and those computed by the reduced-order models.

In Figure 12a the error in computing temperature at critical location A, corresponding to the worst computed case, is plotted for both the reduction methods. As expected after the tuning procedure performed as previously noted, the error is larger if the 1st method is employed. Besides, as expected, the error reduces as the system tends towards steady-state condition if the 1st method is employed, while it tends to be a value different from zero if the 2 nd method is used.

In Figure $12 \mathrm{~b}$ the rate of change of temperature at critical location $\mathrm{A}$ is also plotted. For both methods the error in the rate of change of temperature is characterized by peaks at the beginning of the ramps of upstream fluid temperatures, i.e., at the beginning of thermal transients.
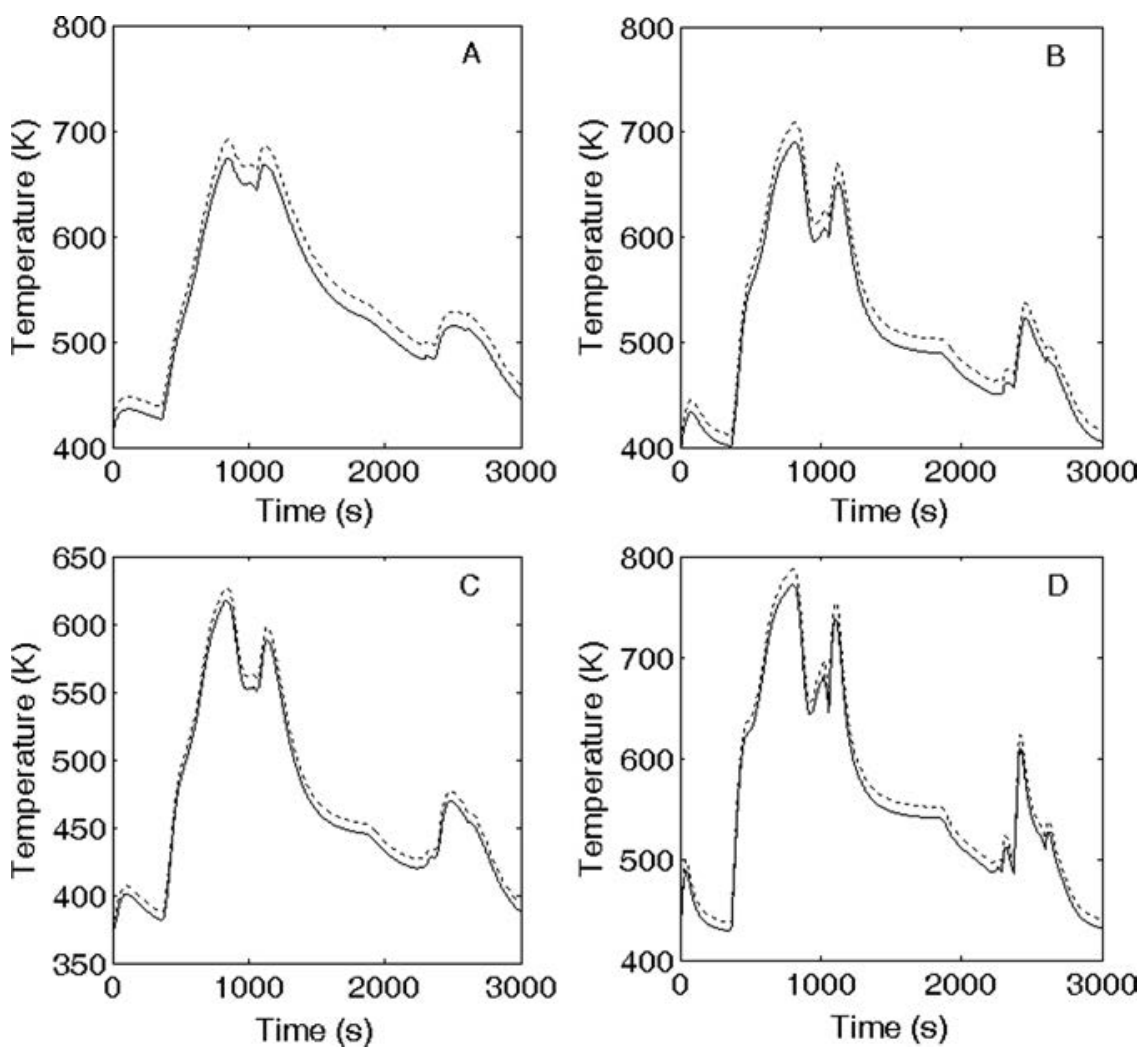

Figure 11 Time history of temperature at critical locations during "real mission." Comparison between full FE model (dotted lines) and reduced model (full lines) with 50 eigenvectors of omitted DOFs: second approach. 

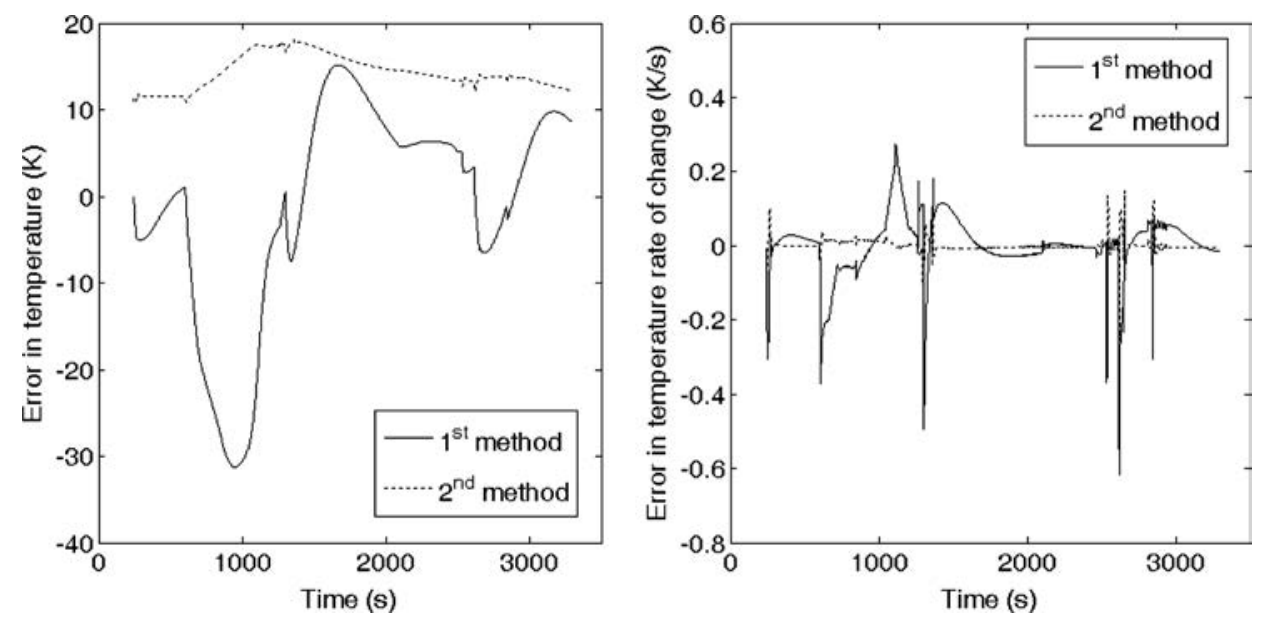

Figure 12 Error in temperature and in temperature rate of change during "real mission" at critical location A.

If the second method is implemented such peaks vanish very quickly and the temperature rate of change is simulated very accurately for the most part of the transients. The behavior is different if the 1 st method is implemented. In this case the error in temperature rate of change is significant during the whole transient and starts vanishing as steady-state conditions are approaching. CPU time to solve the real mission (about 3000 seconds) is shown in Figure 13: both methods take less than 45 seconds to solve the whole mission. With the same hardware the full FE model solution takes more than 3 hours.

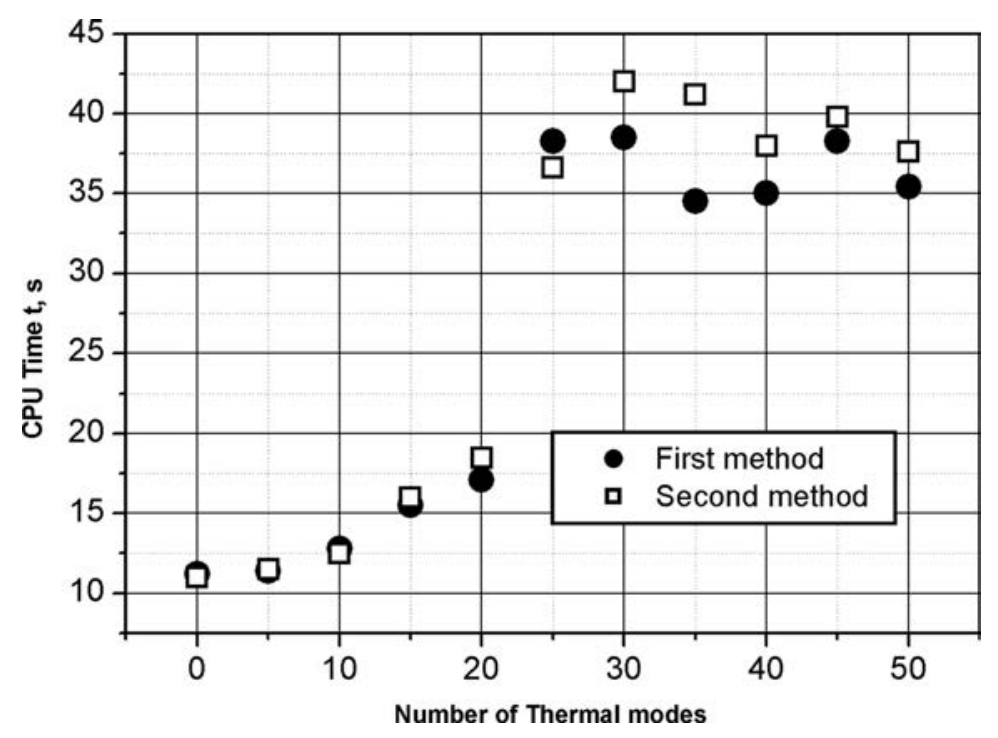

Figure 13 CPU times vs number of thermal modes. 


\section{CONCLUSIONS}

Two methods have been presented in order to obtain reduced-order models for coupled solid/fluid FE thermal models in order to perform fast calculation of thermal transients in critical locations of structural components.

A typical engineering application of the proposed methodologies is the on-line calculation of temperatures performed in both nuclear and aeronautical applications to assess fatigue damage accumulation and residual life.

Both techniques are based on Guyan reduction and Component Mode Synthesis, originally developed in structural dynamics. Both the resulting reducedorder models are characterized by a set of physical temperatures corresponding to locations where temperature has to be computed and by a set of generalized temperatures, namely thermal eigenvectors, introduced to improve the accuracy of the calculation. The inputs for the time integration of the reduced models are the time histories of upstream fluid temperatures of the fluid model surrounding the solid FE thermal model.

The accuracy and the limitations of the methods are tested by means of a study case represented by the coupled FE thermal model of a turbine disk with its secondary air system. The solutions obtained with the reduced-order models are compared to that obtained by a full-scale FE model. The following conclusions are drawn:

1. Both methods allow obtaining reduced-order models suitable for fast calculation of temperature at selected points of a thermal FE model;

2. The first method allows evaluating exactly steady-state temperatures; the addition of eigenvectors allows improving the accuracy of the reduced-order model but the solution does not tend towards that obtained with the full-scale FE model;

3. The second method does not allow evaluating exactly steady-state temperatures; the addition of eigenvectors allows improving the accuracy of the reduced-order model in both steady-states and transients. The solution tends towards that obtained with the full-scale FE model;

4. The size of the reduced-order model suitable for accurate calculation cannot be determined a priori. It is necessary to tune the reduced-order models by means of a reference mission characterised by transients similar to those typical of the real missions.

\section{REFERENCES}

1. J. Broede and H. Pfoertner, Advanced Algorithms Design and Implementation in Onboard Microprocessor Systems for Engine Life Usage Monitoring, 15th Symposium AIMS, Aachen, 1989.

2. M. Koehl, On-Board Engine Life Usage Monitoring by Real Time Computation, Proceedings of the 21st Symposium of the International Committee on Aeronautical Fatigue, Toulouse, 2001.

3. E. Suarez, M. Duffy, and D. Seto, Advanced Life Prediction Systems for Gas Turbine Engines, 39th AIAA/ASME/SAE/ASEE Joint Propulsion Conference and Exhibit, Huntsville, Alabama, July 20-23, 2003.

4. N. K. Mukhopadhyay, B. K. Dutta, H. S. Kushwaha, S. C. Mahajan, and A. Kakodkar, On-Line Fatigue Life Monitoring Methodology for Power Plant Components. Int. J. Press. Vessels Piping, vol. 60, pp. 297-306, 1994. 
5. O. Maekawa, Y. Kanazawa, Y. Takahashi, and M. Tani, Operating Data Monitoring and Fatigue Evaluation Systems and Findings for Boiling Water Reactors in Japan. Nucl. Eng. Des., vol. 15, pp. 135-143, 1995.

6. K. Sakai, K. Hojo, A. Kato, and R. Umehara, On-Line Fatigue Monitoring System for Nuclear Power Plant. Nucl. Eng. Des., vol. 153, pp. 19-25, 1995.

7. S. Zucca, D. Botto, and M. M. Gola, Faster On-Line Calculation of Thermal Stress by Time Integration, Int. J. Press. Vess. Piping, vol. 81, no. 5, pp. 381-387, 2004.

8. D. Petit, R. Hachette, and D. Veyret, A Modal Identification Method to Reduce a High-Order Model: Application to Heat Conduction Modelling, Int. J. Model. Simul., vol. 17, p. 242, 1997.

9. S. Zucca, Identification of a Low-Order Model for Thermal Stress Monitoring, J. Therm. Stresses, vol. 28; pp. 1-15, 2005.

10. R. J. Guyan, Reduction of Stiffness and Mass Matrices, AIAA J., vol. 24, p. 380, 1965.

11. R. R. Craig and M. C. C. Bampton, Coupling of Substructures for Dynamic Analyses, AIAA J., vol. 6, pp. 1313-1319, 1968.

12. D. Botto, S. Zucca, M. M. Gola, E. Troncarelli, and G. Pasquero, Component Modes Synthesis Applied to a Thermal Transient Analysis of a Turbine Disk, Worldwide Aerospace Conference and Technology Showcase, Toulouse, 2002.

13. D. Botto, S. Zucca, M. M. Gola, and S. Salvano, A Method for on Line Temperature Calculation of Aircraft Engine Turbine Discs, ASME Turbo Expo 2002, Amsterdam, vol. 1, pp. 49-55, 2002.

14. D. Botto, S. Zucca, M. M. Gola, A Methodology for On-Line Calculation of Temperature and Thermal Stress Under Non-Linear Boundary Conditions. Int. J. Press. Vess. Pip., vol. 80, no. 1, pp. 21-29, 2003. 